potential applications in the field of diagnosis and vaccination. The use of monoclonal antibodies in research and survey laboratories is now widespread concerning viruses of the classical and African swine fever, Aujeszky's disease, Transmissible gastroenteritis and is extending to bacteriology (Salmonella, Mycoplasma). Nucleic acid probes, presently used as research tools, might also be applied to routine detection of infectious agents. Genetic engineering has allowed the development of several new strategies for immunoprophylaxis : synthetic peptides, recombinant antigens, non-pathogenic live vectors, attenuation by gene deletion. So far, the only commercialised recombinant vaccine is against $\mathrm{E}$. coli-enterotoxicosis in the piglet. Designing of recombinant attenuated strains of pseudorabies virus is in rapid progress. The possibility to differentiate vaccinated from infected animals will be one of the major advantages of the future vaccines.

\title{
Development of an Elisa test to differentiate vaccinated from pseudorabies infected animals
}

\author{
M. ELOIT $^{(1)}$, D. FARGEAUD ${ }^{(2)}$, Ph. VANNIER ${ }^{(3)}$, B. TOMA ${ }^{(1)}$ \\ (1) Ecole Nationale Vétérinaire d'Alfort, Chaire des Maladies contagieuses, \\ 7, avenue du Général-de-Gaulle, 94704 Maisons-Alfort Cedex \\ (2) Rhône Mérieux, I.F.F.A. Mérieux, 254, rue Marcel-Mérieux, 69007 Lyon \\ (3) Ministère de l'Agriculture, Station de Pathologie Porcine, B.P. 9, 22440 Ploufragan
}

The use of two monoclonal antibodies specific of glycoprotein GI and GIII of pseudorabies virus (PRV) led to the development of a competitive Elisa test allowing to differentiate animals infected with PRV from those vaccinated with strains Bartha, NIA4 or Norden. A post-vaccinal serologic response was detectable three to four weeks after vaccination. After virulent challenge of these vaccinated pigs, an infectious serologic response was revealed two weeks after the challenge.

\section{Vaccination trials using the dermic route in the pig}

\author{
A. BRUN ${ }^{(1)}$, Ph. VANNIER ${ }^{(2)}$ \\ (1) Rhône Mérieux, Laboratoire I.F.F.A., 254, rue Marcel-Mérieux, \\ B.P. 700969732 Lyon Cedex 07
}

(2) Station de Pathologie Porcine, B.P. 9, 22440 Ploufragan

Vaccination is necessary to prevent certain diseases in fattening pigs. Vaccination conditions are often difficult and some vaccines are badly tolerated when injected via the intramuscular route. For that reason, vaccination trials were carried out in the pig using the dermic route. Two vaccines against Aujeszky's disease were used : a live modified virus vaccine and an inactivated vaccine prepared from viral glycoprotein. Both vaccines were tested in pigs vaccinated via the dermic route, then challenged with an Aujeszky's disease virus. Immunity results are presented and discussed. 